\title{
Optimización de sistemas de bombeo fotovoltaico a depósitos con criterio técnico-económico.
}

\author{
Carricondo-Antón, $\mathrm{JM}^{1}{ }^{1}$, Jiménez-Bello, $\mathrm{MA}^{2}{ }^{2}$, Manzano Juárez, $\mathrm{J}^{1}$., Royuela Tomas, A $^{1}$, González Altozano, $\mathrm{P}^{3}$.
}

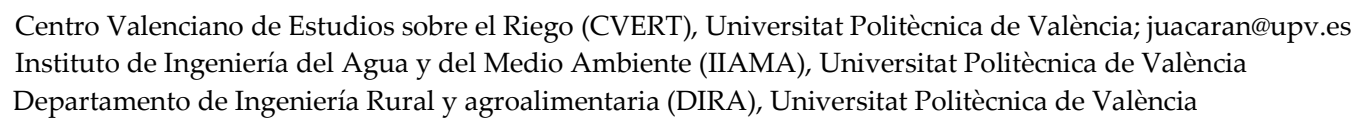

Resumen: Para el cálculo de instalaciones donde hay bombeos de agua entre dos depósitos se suele diseñar las instalaciones de bombeo fotovoltaico (FV) siguiendo las pautas de instalaciones tradicionales. Se calcula el punto de funcionamiento y se selecciona una bomba que su curva (Hm-Q) sea lo más próxima al punto de funcionamiento. El problema de esta metodología es que parte de la energía solar del día se desperdicia y podría ser utilizada para el bombeo de agua. Por ello es importante la selección del tipo y número de bombas que pueden trabajar en paralelo. Este trabajo tiene como objetivo principal desarrollar una metodología para la optimización del número de bombas que pueden trabajar en paralelo y del tamaño del generador en instalaciones de riego FV a balsas. El caso de estudio consiste en un bombeo entre dos depósitos, donde él de mayor cota abastece por gravedad una superficie de riego de 42.21 ha, en la comunidad de regantes Sector 4, próximo al municipio de Picassent perteneciente a la Comunidad Valenciana situada en el Este de España. La metodología empleada se basa en el diseño de la instalación FV (Potencia pico $(\mathrm{Pp}, \mathrm{W})$ ) y del equipo de bombeo mediante la metodología estándar. Se realiza un planteamiento de las hipótesis de bombeo con diferente número de bombas funcionando en paralelo (BFP) (de 1 a 8 bombas) donde la suma de caudales de todas las BFP de las diferentes hipótesis será igual al caudal de la hipótesis de 1 bomba. El volumen bombeado anual (VBA, $\mathrm{m}^{3} \mathrm{anno}^{-1}$ ) se calcula determinando las horas de potencia disponible $(\mathrm{HPD}, \mathrm{h}$ ) para cada hipótesis (donde Potencia neta $($ Pnet, $\mathrm{W})>$ Potencia requerida $\left.\left(\mathrm{P}_{\mathrm{b}}, \mathrm{W}\right)\right)$ para todos los días del año para conocer la variación del VBA. Seguidamente, se utilizan distintas hipótesis de factor potencia (FP) (Cociente entre $\mathrm{Pp}$ y $\mathrm{Pb}$ ) con el fin de estudiar el mínimo número de placas solares requeridas. Por último, se selecciona el número de bombas por criterio económico. Como principales resultados se obtiene un mayor aprovechamiento de la irradiancia, para la hipótesis con 1 bomba la potencia mínima requerida para que arranque la misma es de $24,4 \mathrm{~kW}$, lo que equivale a un umbral de irradiancia de 336,2 $\mathrm{Wm}^{-2}$ para el mes más desfavorable. Sin embargo, para que comience el bombeo con la hipótesis de $5 \mathrm{BFP}$, solo se requiere de 4,9 $\mathrm{kW}$, siendo el umbral de irradiancia, para el mes más desfavorable es de 65,2 $\mathrm{Wm}^{-2}$. Al aumentar el número de bombas se aumenta el VBA hasta casi un 27\%, y viendo los incrementos de bombeo a lo largo de este año en relación con las necesidades de volumen a bombear se determina que para el número óptimo de bombas que sería 5, en este caso de estudio, el FP se disminuye en un 50,7\%. La función económica nos indica el número de BFP a seleccionar, siendo la hipótesis de 5 bombas la que menor coste tiene.

Palabras clave: Bombeo fotovoltaico; Selección de bombas; Optimización riego fotovoltaico 


\title{
XXXVIII Congreso Nacional de Riegos
} CARTAGENA 2021

\section{Optimization of photovoltaic tank pumping systems with technical-economic criteria.}

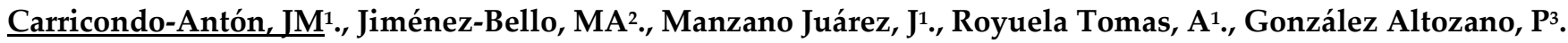

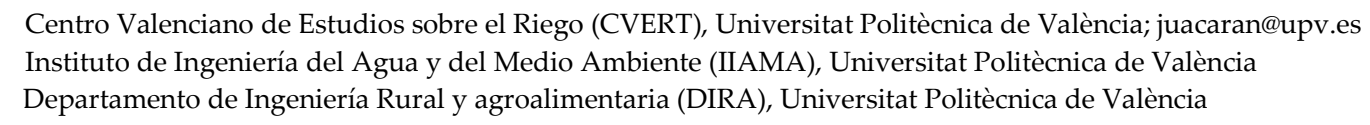

Abstract For the calculation of installations where there are water pumps between two tanks, photovoltaic (PV) pumping installations are usually designed following the guidelines of traditional installations. The operating point is calculated and a pump is selected whose curve (Hm-Q) is closest to the operating point. The problem with this methodology is that part of the day's solar energy is wasted and could be used for pumping water. For this reason, it is important to select the type and number of pumps that can work in parallel. The main objective of this work is to develop a methodology for the optimization of the number of pumps that can work in parallel and the size of the generator in PV irrigation pond irrigation installations. The case study consists of a pumping between two reservoirs, where the higher level one supplies by gravity an irrigation surface of 42.21 ha, in the Sector 4 irrigation community, close to the municipality of Picassent belonging to the Valencian Community located in the East from Spain. The methodology used is based on the design of the PV installation (Peak Power $(\mathrm{Pp}, \mathrm{W}))$ and the pumping equipment using the standard methodology. A proposal is made of the pumping hypotheses with different number of pumps operating in parallel (BFP) (from 1 to 8 pumps) where the sum of the flows of all the BFPs of the different hypotheses will be equal to the flow of the hypothesis of 1 pump. The annual pumped volume (VBA, m3year-1) is calculated by determining the hours of available power (HPD, h) for each hypothesis (where Net power (Pnet, W) $>$ Required power $(\mathrm{Pb}, \mathrm{W}))$ for all the days of the year to know the variation of the VBA. Next, different hypotheses of power factor (FP) (quotient between $\mathrm{Pp}$ and $\mathrm{Pb}$ ) are used in order to study the minimum number of solar panels required. Finally, the number of pumps is selected by economic criteria. As main results, a greater use of irradiance is obtained, for the hypothesis with 1 pump the minimum power required for it to start is $24.4 \mathrm{~kW}$, which is equivalent to an irradiance threshold of $336.2 \mathrm{Wm}^{-2}$ for the worst month. However, to start pumping with the $5 \mathrm{BFP}$ hypothesis, only $4.9 \mathrm{~kW}$ is required, the irradiance threshold being $65.2 \mathrm{Wm}-2$ for the most unfavourable month. By increasing the number of pumps, the VBA is increased to almost $27 \%$, and looking at the pumping increases throughout this year in relation to the volume needs to be pumped, it is determined that for the optimal number of pumps that would be 5 , in this case study, the FP is decreased by $50.7 \%$. The economic function indicates the number of BFP to select, being the hypothesis of 5 pumps the one with the lowest cost.

Keywords: Photovoltaic pumping; Selection of pumps; Photovoltaic irrigation optimization 


\section{Congreso Nacional de Riegos CARTAGENA 2021}

\section{1. introducción}

Los sistemas fotovoltaicos son susceptibles de aplicación para el riego solar como muestra la bibliografía [1-3]. Por lo general, para el cálculo de instalaciones donde hay bombeos de agua entre dos depósitos, en el caso de bombeo fotovoltaico, se dimensionan determinando el punto de funcionamiento y seleccionando una bomba cuya curva (Hm-Q) pase lo más cerca del mismo y con el mejor rendimiento posible. El problema de esta metodología es que durante buena parte del día la energía solar es insuficiente para mantener o bien poner en marcha la bomba y se desperdicia. Cabe la posibilidad de poner, en lugar de una única bomba, varias bombas más pequeñas conectadas en paralelo que puedan aprovechar la energía disponible con menores niveles de radiación para una misma potencia de generador fotovoltaico instalada.

El objetivo de este trabajo es proponer una metodología de dimensionado con la que llegar a establecer, tanto el número de bombas en paralelo, como la potencia del generador fotovoltaico necesario, para el funcionamiento adecuado de una impulsión a una balsa.

\section{Materiales y métodos}

\subsection{Zona de estudio}

Este estudio se ha realizado en una pequeña zona de la Comunidad de Regantes Picassent Sector 4 cercana al municipio de Picassent en la Comunidad Valenciana, en el este de España. La instalación abastece una superficie de 42,21 ha, que se riegan desde un depósito elevado de $1680 \mathrm{~m}^{3}$, situado junto al cabezal de San Rafael (coordenadas 39 22'51,46"N, $0^{\circ} 31^{\prime} 59,43^{\prime \prime} \mathrm{O}$ ), siendo la cota de su lamina libre de agua de 165.3 m.s.n.m. Esta cota permite efectuar el riego de esta zona sin necesidad de energía adicional. Este depósito es alimentado, desde el depósito del cabezal Sagrada Familia siendo la cota de su lamina libre de 142 m.s.n.m (coordenadas 39²3'13,06"N, $0^{\circ} 31^{\prime} 12,65 " \mathrm{O}$ ), también perteneciente a la comunidad de regantes. La instalación hidráulica está compuesta por una conducción de $1400 \mathrm{~m}$ de longitud de PVC de DN 400 y PN6 la cual une los dos depósitos. En la instalación se proyectó y selecciono una única bomba que requiere de una potencia de $24.4 \mathrm{~kW}$, abastecida mediante una instalación fotovoltaica compuesta de 170 paneles de $320 \mathrm{Wp}$ generando una potencia pico de $54.4 \mathrm{kWp}$ con un factor o relación Potencia generada-Potencia requerida de 2,23.

\subsection{Metodología aplicada}

En este trabajo proponemos una metodología que permita determinar tanto el número de bombas más adecuado como la potencia del generador, que garantice el suministro hídrico necesario y minimice la potencia fotovoltaica instalada.

\subsubsection{Selección de generador fotovoltaico y bomba hidráulica}

Se considera el mes más desfavorable (MUM) aquel en que la relación obtenida con la ecuación (1) es mayor.

$$
M U M_{i}=\frac{E H_{i}}{\operatorname{Gmax}_{i}}
$$

Donde $E H_{i}$ es la energía hidráulica promedio diaria mensual $(\mathrm{kWh})$ y $\operatorname{Gmax}_{i}$ es la radiación solar disponible promedio diaria mensual $(\mathrm{kWh})$. La radiación $\left(\operatorname{Gmax}_{i}\right)$ se ha obtenido a partir de los datos de irradiancia de un 


\section{Congreso Nacional de Riegos CARTAGENA 2021}

Año Meteorológico Tipo proporcionado por el Sistema de información geográfica fotovoltaica (PVGIS) del servicio de ciencia y conocimiento de la Comisión Europea (https://ec.europa.eu/jrc/en/pvgis) para la ubicación de la instalación. EHi se determina, para cada mes del año, con la expresión (2):

$$
E H_{i}=\frac{\rho \cdot g \cdot H m \cdot V}{3600}
$$

Donde $\rho$ es la densidad del agua $\left(\mathrm{kg} . \mathrm{m}^{-3}\right), g$ es la aceleración de la gravedad $\left(9.81 \mathrm{~m} \mathrm{~s}^{-2}\right), \mathrm{Hm}$ es la altura manométrica $(\mathrm{m}), V_{\text {dia }}$ es el volumen de agua a bombear diariamente $\left(\mathrm{m}^{3} \mathrm{dia}^{-1}\right)$.

Para el cálculo del volumen a bombear en cada mes usamos la siguiente ecuación:

$$
V_{\text {day }}=10^{-3} \cdot E T o \cdot K c \cdot f c_{\text {month }} \cdot \text { Area }
$$

Donde $V_{\text {day }}$ es volumen bombeado diariamente $\left(\mathrm{m}^{3} \mathrm{dia}^{-1}\right)$, ETo es la evapotranspiración de referencia (mm), Kc es el coeficiente de cultivo y $f c_{\text {month }}$ es el factor de corrección mensual propuestos para cítricos por el Institut Valencià d'Investigacions Agràries (IVIA) (www.ivia.gva.es), Area es la superficie a regar (42.21 ha).

Teniendo en cuenta las características de la impulsión, la altura manométrica (Hm) que debe suministrar el sistema de bombeo, se determina:

$$
\begin{aligned}
& H m=\left(Z_{2}-Z_{1}\right)+h r+h s \\
& h r=10.674 \cdot \frac{Q^{1.852}}{C^{1.852} \cdot D^{4.78}} \cdot L
\end{aligned}
$$

Donde $\left(Z_{2}-Z_{1}\right)$ es la diferencia de cotas $(23,3 \mathrm{~m}), h r$ son las pérdidas de carga por rozamiento determinadas según Hazen-Williams (m) (eq 5), $h s$ son las pérdidas de carga localizadas (m), $Q$ es el caudal de diseño para cada mes $\left(\mathrm{m}^{3} \mathrm{~s}^{-1}\right)$ a partir del volumen y las horas solar pico, $C$ es el coeficiente de rugosidad (en PVC, C = 150), $D$ el diámetro interno (380.4 m) y $L$ la longitud de la tubería de impulsión (1400 m).

Para el mes más desfavorable, el caudal de diseño $\left(\mathrm{Q}, \mathrm{m}^{-3} \mathrm{~s}^{-1}\right)$ se determina teniendo en cuenta que las horas de funcionamiento de la bomba ( $\mathrm{PH}$ ) son menores a las horas solar pico (HSP) para cada mes. HSP se ha calculado promediando las irradiancias diarias de cada mes $\left(\mathrm{kWh} \cdot \mathrm{m}^{-2} \cdot \mathrm{dia}^{-1}\right)$. $\mathrm{PH}$ se ha determinado sumando las horas en que la potencia requerida por las bombas es menor a la potencia generada por el generador fotovoltaico.

$$
Q=\frac{V_{d a y}}{P H}
$$

A partir de la Energía Hidráulica $\left(E H_{i}\right)$ para el mes más desfavorable, se determina la Energía Eléctrica (EE) (Eq.7), la potencia pico necesaria $\left(P_{p}\right)$ (Eq.8) y finalmente el número de paneles ( $\left.\mathrm{N}_{\text {paneles}}\right)$ (Eq.9) mediante las siguientes ecuaciones:

$$
\begin{gathered}
E E=\frac{E H_{i}}{\eta_{m b}} \\
P_{p}=\frac{E E}{P R \cdot H S P} \\
N_{\text {paneles }}=\frac{P_{p}}{P_{p_{\text {panel }}}}
\end{gathered}
$$

Siendo $\eta_{m b}$ el rendimiento del grupo motobomba y PR el rendimiento energético del sistema.

Para el cálculo del número de paneles solares (Eq.9) se escoge un panel de $320 \mathrm{Wp}$, se considera un PR = 0.4 , y un rendimiento del grupo moto-bomba de $\eta_{m b}=0.8$ 


\section{Congreso Nacional de Riegos CARTAGENA 2021}

\subsubsection{Potencia neta del generador}

En la determinación de la potencia del generador se considera el efecto de la radiación y la temperatura sobre el mismo, estos datos se trataron con una frecuencia horaria para todos los días del periodo de estudio. Los datos meteorológicos empleados se obtienen, con una frecuencia horaria de la estación agroclimática de Picassent del IVIA, perteneciente al Sistema de Información Agroclimática para el Regadío (SIAR). La temperatura de la célula (Tcel) se determina utilizando la expresión de [4]:

$$
\text { Tcel }=T+\frac{(T O N C-20) \cdot G}{800}
$$

Donde T es Temperatura en grados centígrados, TONC es la temperatura nominal de funcionamiento de la célula fotovoltaica (en este caso $45^{\circ} \mathrm{C}$ ) y $\mathrm{G}$ es la irradiancia $\mathrm{Wm}^{-2}$.

El sistema FV está formado por 170 paneles con 72 células FV de 6 pulgadas de material policristalino con una potencia de $320 \mathrm{~W}$. La potencia fotovoltaica instantánea neta disponible se determina mediante la ecuación:

$$
P_{\text {int }}=P_{p} \cdot \frac{G}{1000} \cdot\left(1+\frac{\alpha_{p}}{100} \cdot(\text { Tcel }-25)\right)
$$

Donde Pp es la potencia pico de la placa solar, G es la irradiancia $\mathrm{Wm}^{-2}$ para el año 2019, $\alpha_{p}$ es el coeficiente de variación de $\mathrm{Pp}$ con la temperatura $\left(\%{ }^{0} \mathrm{C}^{-1}\right)$ y $\mathrm{Tcel}$ es la temperatura de la célula fotovoltaica.

Por último, se debe de tener en cuenta que la potencia neta (Pnet) que las bombas pueden utilizar, se ve afectada por la eficiencia del inversor y del motor del equipo.

$$
P_{\text {net }}=P_{\text {int }} \cdot \eta_{f c} \cdot \eta_{m b}
$$

Donde $\eta_{f c}$ es la eficiencia del inversor que se considera $0.9 \mathrm{y}$ un rendimiento del grupo moto-bomba que se considera $\eta_{m b}=0.8$.

\subsection{Hipótesis de bombeo}

Para estudiar el efecto que tiene el aumento del número de bombas en paralelo en el caudal bombeado, se plantean 8 hipótesis. La hipótesis de partida con una bomba y las hipótesis 2 a 8 con un número creciente de bombas. La primera considera la bomba que sería necesaria para elevar un Q de $280 \mathrm{~m}^{3} \mathrm{~h}^{-1}$ a una $\mathrm{Hm}$ de $25.1 \mathrm{~m}$, esta sería el caso de utilizar una única bomba. La segunda hipótesis estaría formada por dos bombas de la mitad de $Q$ funcionando en paralelo, la tercera por tres bombas de un tercio del $Q$, y así sucesivamente, hasta llegar a la octava en que participan hasta 8 bombas. Las bombas consideradas son de la marca comercial Indar (https://www.ingeteam.com) para seleccionar la misma marca comercial de la instalación. El funcionamiento de las mismas se ha simulado considerando los modelos de las bombas comerciales. La selección de las bombas con los diferentes puntos de funcionamiento, según el número de bombas estudiadas, se realiza en base a la máxima eficiencia que pueden proporcionar los modelos comerciales.

En cada hipótesis, en función de la energía disponible, se irán conectando bombas hasta alcanzar la máxima energía requerida. Por ejemplo, cuando la energía requerida por una bomba para su funcionamiento a velocidad nominal sea menor que la energía suministrada por el sistema fotovoltaico esa bomba comenzara a impulsar el caudal correspondiente a la potencia transmitida al eje. Por ello, las HPD para cada bomba de cada hipótesis se producirán cuando Pnet $>\mathrm{P}_{\mathrm{b}_{\mathrm{i}}}$, siendo el caudal impulsado la suma de los caudales bombeados por las bombas de cada hipótesis. Cuando la energía suministrada por las placas solares sea superior a la requerida por dos bombas, se conectará la segunda bomba y suministrando el caudal de esas 2 bombas y así sucesivamente hasta alcanzar el número máximo de bombas de cada hipótesis. El caudal y altura manométrica de cada bomba dentro de cada 


\section{Congreso Nacional de Riegos CARTAGENA 2021}

hipótesis se calculan según la Eq.13. La Hm mínima es de $23.3 \mathrm{~m}$ con un caudal de $35 \mathrm{~m}^{3} \mathrm{~h}^{-1} \mathrm{y}$ se da en la hipótesis de 8 bombas, la Hm máxima es de $25.1 \mathrm{~m}$ y se producirá cuando se impulsan $280 \mathrm{~m}^{3} \mathrm{~h}^{-1}$ para todas las hipótesis.

$$
Q_{B^{\prime o m b a}}=\frac{Q_{I P}}{N}
$$

Donde $Q_{B o m b a}$ es el caudal que suministran las bombas de cada hipótesis en funcionamiento $\left(\mathrm{m}^{3} \mathrm{~h}^{-1}\right), Q_{I P}$ es el caudal que debería suministrar si se instalara una única bomba $\left(280 . \mathrm{m}^{3} \mathrm{~h}^{-1}\right)$ y $N$ es el número de bombas que se considera en cada una de las hipótesis (de 1 a 8 ).

Con la variación del caudal impulsado varían las pérdidas de carga, y se modifica la altura manométrica y la eficiencia de cada bomba y con ello la potencia requerida por la misma $\left(P_{b_{i}}\right)$.

$$
\mathrm{P}_{\mathrm{b}_{\mathrm{i}}}=\frac{1}{\eta_{\mathrm{i}}} \cdot \gamma \cdot \mathrm{Q}_{\mathrm{i}} \cdot \mathrm{Hm}_{\mathrm{i}}
$$

Donde $\eta_{i}$ es la eficiencia de las bombas para cada número de bombas de cada hipótesis, $\gamma$ es el peso específico del agua $\left(\mathrm{Nm}^{-3}\right), \mathrm{Q}_{i}$ es el caudal requerido para cada número de bombas de cada hipótesis $\left(\mathrm{m}^{3} \mathrm{~s}^{-1}\right)$ y $\mathrm{Hm}_{i}$ es la altura manométrica requerida para cada número de bombas de cada hipótesis (m).

El volumen semanal bombeado $\left(V_{a n ̃ o}\right)$ se calcula mediante la siguiente ecuación:

$$
V_{a \tilde{n} o}=\sum\left(I W h_{B o m b a_{i}} \cdot Q_{B_{o m b a}}\right)
$$

Siendo $V_{a n ̃ o}$ el volumen en $\mathrm{m}^{3}$ anual, $I W h_{B o m b a_{i}}$ son las horas anuales en que la potencia suministrada al número de bombas de cada hipótesis es superior a la requerida.

\subsection{Generador fotovoltaico}

El generador de partida cuenta con un número de paneles, y el Factor de Potencia (Fp) que relaciona la ponencia instalada frente a potencia requerida:

$$
F p=\frac{N_{\text {paneles }} \cdot P P_{p}}{P_{b_{i}}}
$$

Con objeto de estudiar el efecto del tamaño del generador en el funcionamiento de la instalación y en el bombeo de agua se han considerado diferentes relaciones de Fp, siendo estas $0.5,1,1.1,1.2,1.3,1.4,1.5,1.6,1.7$, $1.8,1.9,2,2.5,3,4,5,10$. Además se estudia el Fp instalado (170 paneles fotovoltaicos) con la hipótesis de 1 bomba (Fp: 2.23), de 3 bombas (Fp: 2.1), de 5 bombas (Fp: 2.06) y de 8 bombas (Fp: 1.81). Se desarrolla en el apartado 3.2.

\subsection{Selección del número de bombas por criterio económico.}

En este apartado se estudia la variación del coste económico para las diferentes hipótesis, de 1 a 8 bombas, y diferente tamaño de generador. El coste de las bombas para las hipótesis 1, 3, 5 y 8, el cual es obtenido del catálogo comercial de Indar, es de 10,777.2 $€ \mathrm{Ud}^{-1}, 4,600.6 € \mathrm{Ud}^{-1}, 3,293.5 € \mathrm{Ud}^{-1}$ y 2,703.3€ Ud $\mathrm{Ud}^{-1}$ respectivamente. El coste de cada panel fotovoltaico actualmente en la casa Solar Innova es de $124.2 € \mathrm{Ud}^{-1}$. Ambos catálogos son precios del año 2021. El cálculo económico se ha limitado al coste de las bombas y de las placas solares, sin tener en cuenta otros costes involucrados o ingresos como son los excedentes de energía. Por ello, el cálculo del coste económico de cada hipótesis $(\mathrm{CEh})$ se ha realizado mediante la siguiente ecuación, para las hipótesis con 1, 3, 5 y 8 bombas.

$$
C E_{h}=P C_{h} \times P N_{n}+P V C_{h} \times N_{\text {paneles }_{h}}
$$

Donde PCh es el coste del modelo de las bombas de cada hipótesis $\left(€ \mathrm{Ud}^{-1}\right)$, el PVCh es el coste del panel fotovoltaico $\left(€ \mathrm{Ud}^{-1}\right)$ y Npanelesh es el número de paneles fotovoltaicos de cada hipótesis para abastecer el volumen requerido. 


\section{Congreso Nacional de Riegos CARTAGENA 2021}

\section{Resultados y discusión}

\subsection{Variación del volumen impulsado con cada una de las hipótesis.}

En la figura 1 se muestra la evolución del volumen bombeado semanalmente a lo largo del año 2019 para cada una de las hipótesis planteadas. En ella destaca que el volumen impulsado con la hipótesis de partida ( 1 bomba) es significativamente inferior al del resto de hipótesis a todo lo largo del año.

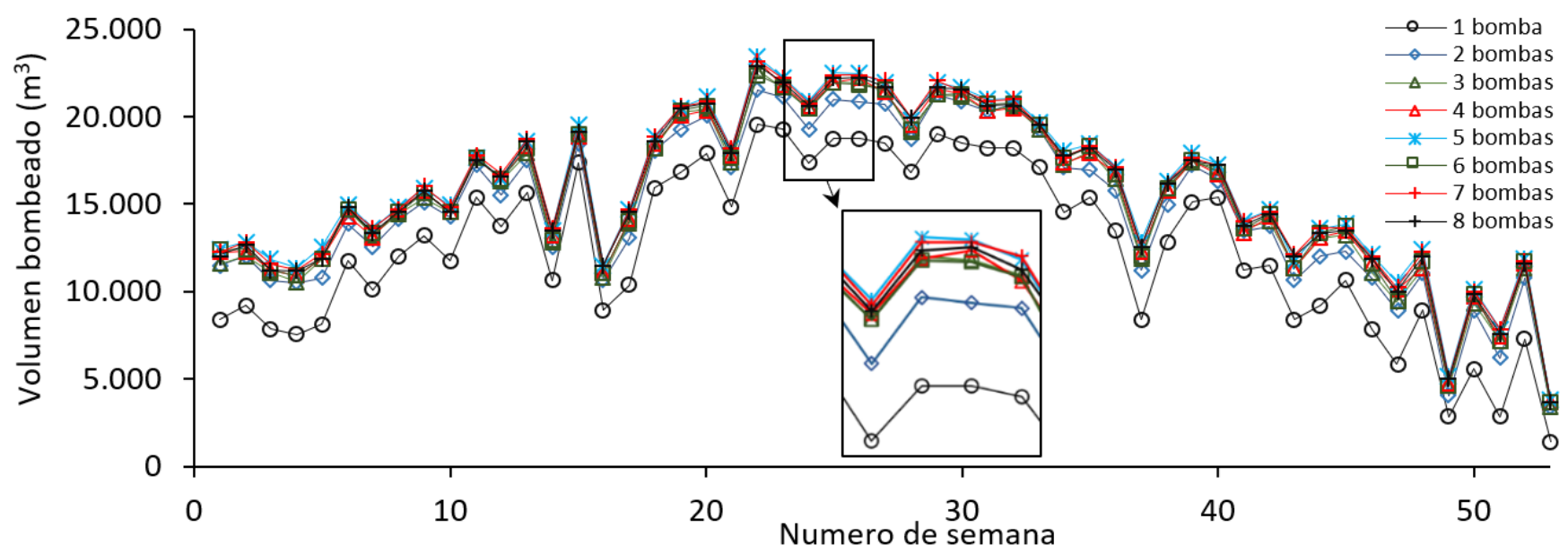

Figura 1: Volumen bombeado $\left(\mathrm{m}^{3}\right)$ para la irradiancia medida $\left(\mathrm{W} \mathrm{m}^{2}\right)$ por semana durante el año 2019.

Al aumentar el número de bombas en paralelo con cada una de las hipótesis aumenta el caudal bombeado, pero las diferencias son cada vez menores. Aunque cada bomba bombea menos caudal que en la hipótesis anterior, aumentan las horas de energía disponible (Energía suministrada-Energía requerida) y con ello el volumen bombeado $\left(\mathrm{m}^{3}\right)$. Este aumento tiene un límite, puesto que conforme aumenta el número de bombas se reduce la ganancia de volumen inyectado respecto de la hipótesis anterior. La ganancia respecto de la hipótesis con 1 bomba crece hasta la hipótesis de 5 bombas con un aumento de un $27 \%$ del volumen anual inyectado respecto de la hipótesis de partida. A partir de la hipótesis con 5 bombas la ganancia deja de crecer y se hace asintótica, lo cual muestra que el sistema de bombeo más adecuado para este caso corresponde a la hipótesis de 5 bombas en paralelo (figura 2).

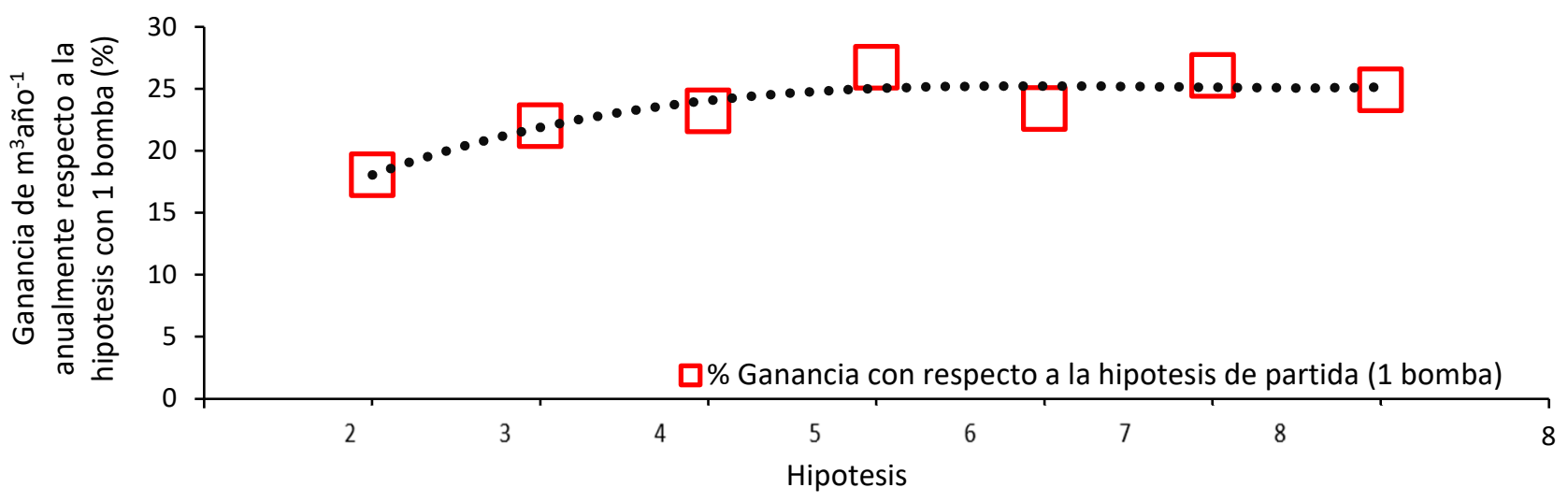

Figura 2: Porcentaje de ganancia de volumen bombeado de las hipótesis de 2 a 8 bombas respecto de la hipótesis de 1 bomba y línea de tendencia de la ganancia. 


\section{Congreso Nacional de Riegos CARTAGENA 2021}

\subsection{Variación del volumen impulsado en función de la potencia del generador fotovoltaico.}

En este apartado se estudió la variación del volumen bombeado anual para las hipótesis con 1, 3, 5 y 8 bombas en función de la potencia pico del generador fotovoltaico.

En la Figura 3 se muestra el aumento en el volumen bombeado $\left(\mathrm{m}^{3} \mathrm{año}^{-1}\right)$ al aumentar el tamaño del generador fotovoltaico para cada una de las hipótesis con 1, 3, 5 y 8 bombas en paralelo. Como era de esperar, al aumentar la potencia del generador fotovoltaico también aumenta el volumen bombeado en todas las hipótesis. Y para una potencia determinada de generador fotovoltaico, el volumen bombeado es tanto mayor cuanto mayor es el número de bombas de la hipótesis. También en esta figura se observa que la ganancia en volumen bombeado es menor conforme las hipótesis tienen mayor número de bombas. De tal forma que al pasar de la hipótesis con 1 bomba a la de 3 bombas, o de 3 a 5 , la ganancia en volumen bombeado es muy importante, pero la diferencia se reduce al pasar de 5 a 8 bombas. El efecto del tamaño del generador es muy importante (pendiente muy acusada) hasta un tamaño de generador que depende del número de bombas de la hipótesis. Se puede observar que conforme aumenta la potencia pico instalada aumenta el volumen anual impulsado. También cuando se aumenta el número de bombas que funcionan en paralelo aumentan los $\mathrm{m}^{3} \mathrm{año}^{-1}$ impulsados, pero los aumentos van disminuyendo conforme se aumenta el número de bombas que funcionan en paralelo siendo la ganancia muy reducida cuando se pasa de la hipótesis de 5 a 8 bombas funcionando en paralelo. Por ello, se vuelve a confirmar qué el óptimo para este caso de estudio sería la instalación de 5 bombas funcionando en paralelo.

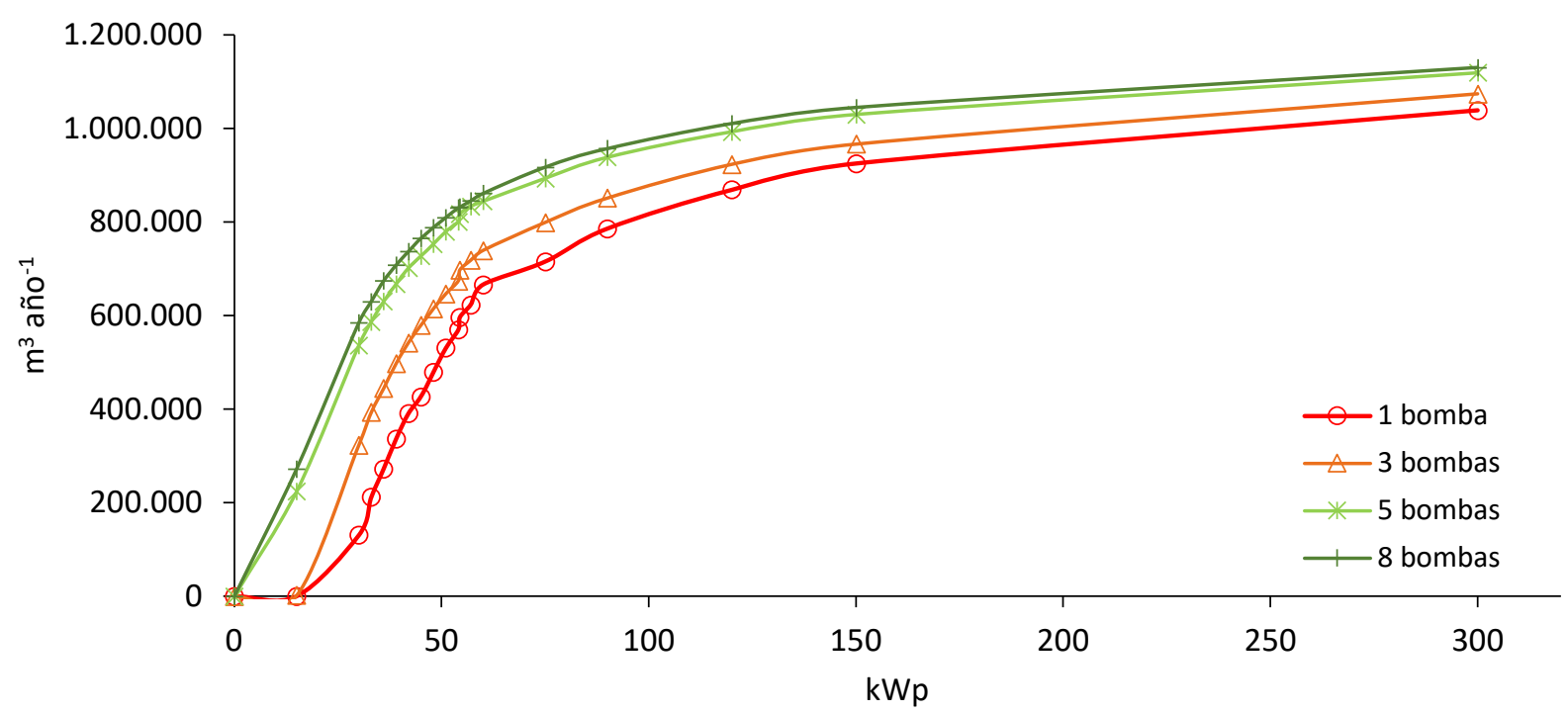

Figura 3: Volumen bombeado $\left(\mathrm{m}^{3}\right)$ frente a los $\mathrm{kWp}$ del generador fotovoltaico para diferentes factores de potencia que relacionan potencia instalada y potencia requerida.

\subsection{Variación del volumen impulsado en función del tamaño del generador FV.}

En este apartado se estudia el Fp o relación mínima que debe haber entre la potencia pico instalada y la potencia requerida por las bombas, en cada una de las hipótesis, para poder suministrar el volumen semanal necesario para abastecer la zona de regadío.

En la Figura 4 se muestra el volumen bombeado semanalmente con las hipótesis de 1, 3, 5 y 8 bombas con capacidad fotovoltaica suficiente para abastecer el volumen semanal requerido por la zona de riego. Como ya vimos en la figura 7 y también se desprende de la figura 8, el tamaño necesario de 


\section{Congreso Nacional de Riegos CARTAGENA 2021}

generador fotovoltaico aumenta conforme se reduce el número de bombas siendo necesario un Fp, o relación, de 2,23 con la hipótesis de 1 sola bomba, de 1,6 con 3 bombas, de 1,1 con 5 bombas y finalmente cuando se instalan 8 bombas sería necesario un Fp de 1. Por otro lado, también se observa que, al aumentar el número de bombas, la diferencia de Fp se va reduciendo ya que de 1 (Fp: 2.23 y $54.4 \mathrm{kWp}$ ) a 3 bombas (Fp: 1.6 y $41.3 \mathrm{kWp}$ ) se reduce el Fp en 0.63, de 3 a 5 bombas (Fp: 1.1 y $28.9 \mathrm{kWp}$ ) se reduce el Fp en 0.5 y de 5 a 8 bombas (Fp: 1.0 y $30 \mathrm{kWp}$ ) se reduce el $\mathrm{Fp}$ en 0.1 , por lo que podemos considerar que a partir de 5 bombas ya no hay reducciones significativas de la potencia fotovoltaica mínima requerida.

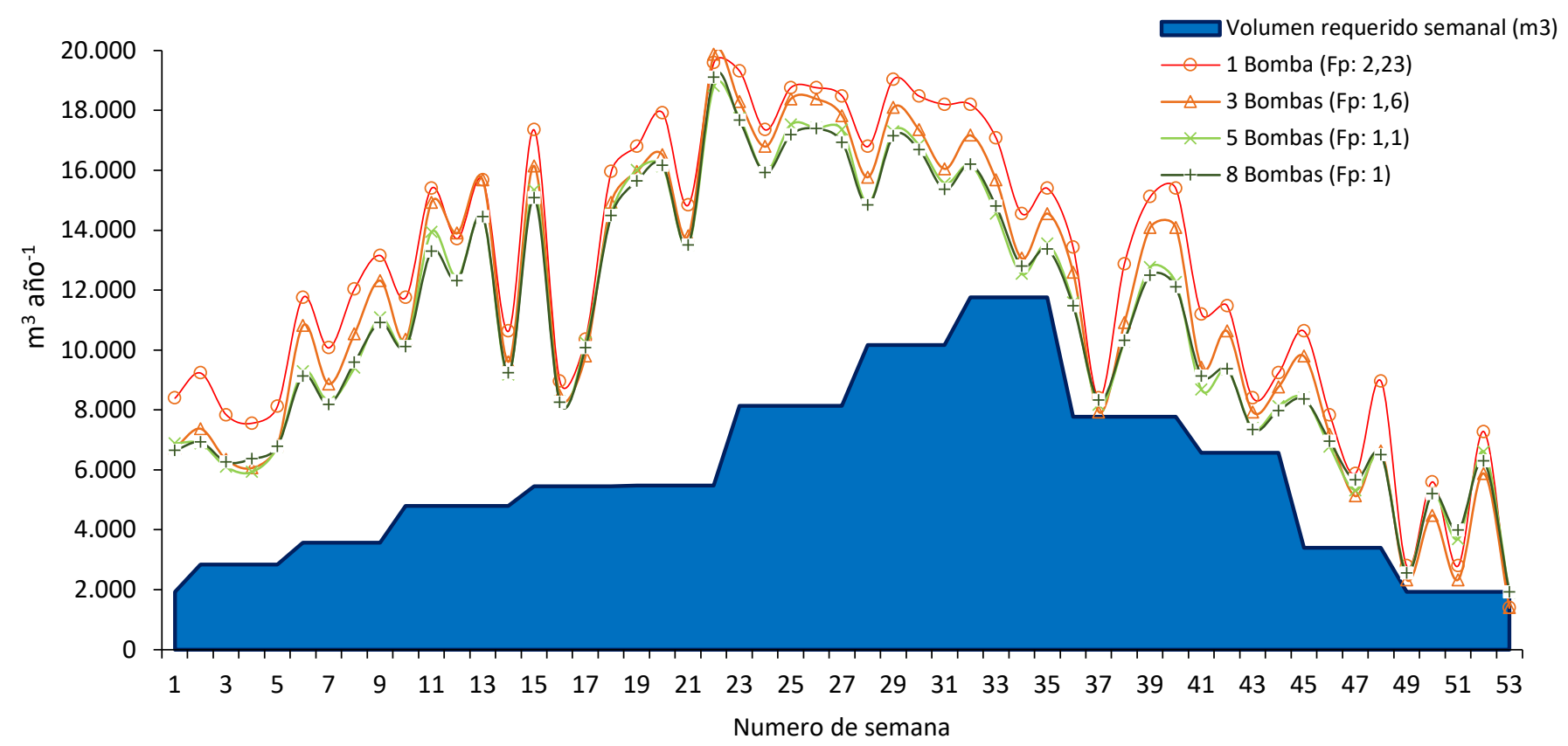

Figura 4: Volumen bombeado semanalmente $\left(\mathrm{m}^{3}\right)$ con las hipótesis de 1, 3, 5 y 8 bombas con factores de potencia 2.23, 1.6, 1.1 y 1 respectivamente para bombear el agua semanal requerida, frente al volumen semanal requerido por la zona de riego.

\subsection{Selección del número de bombas por criterio económico.}

En este apartado se describen los resultados de la selección de bombas por criterio económico como se indica en el apartado 2.5. Como muestra la figura 5 al aumentar el número de bombas se puede disminuir el número de paneles fotovoltaicos para abastecer un determinado volumen e impulsar. Como cabía esperar, al aumentar el número de bombas reduciendo su coste unitario y la reducción del número de paneles fotovoltaicos produzca una reducción del coste de instalación. Sin embargo, la pendiente de la curva comienza a aumentar a partir de la hipótesis de 5 bombas en funcionamiento. Esto es debido a dos causas, la primera es que conforme se disminuye la potencia de la bomba se va reduciendo la diferencia de precio entre las bombas y sin embargo se va aumentando el número de bombas. La segunda causa es que conforme se aumenta el número de bombas estás presentan menores eficiencias y por ello el Fp se va reduciendo, presentando solo una ganancia del 0.1 de la hipótesis de 5 a la de 8 bombas. 


\section{Congreso Nacional de Riegos CARTAGENA 2021}

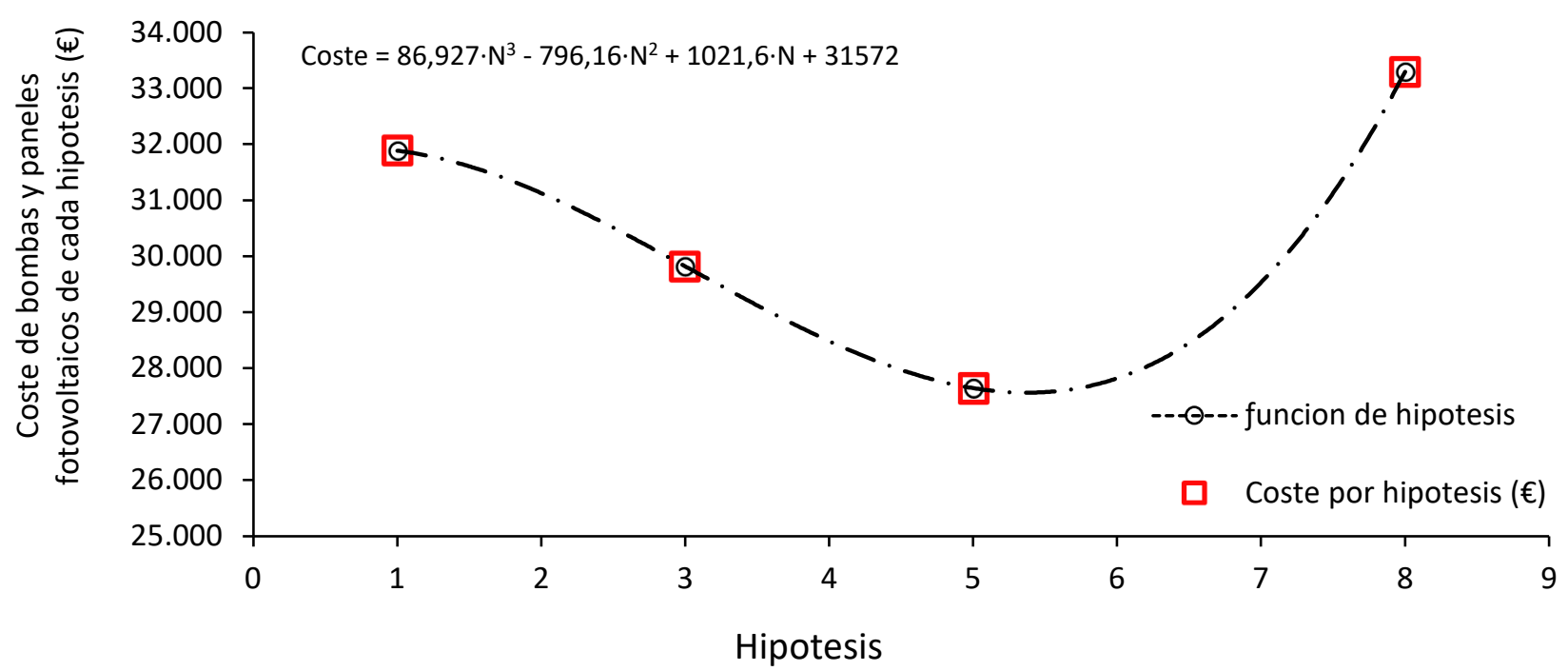

Figura 5: Coste de las bombas y paneles fotovoltaicos que los abastecen las hipótesis de 1, 3, 5 y 8, en función del número de bombas.

Por tanto, también se confirma con el criterio económico que la opción más favorable para este caso es colocar 5 bombas en paralelo. Por último, hay que tener en cuenta que la función se ha limitado al coste de las bombas y de los paneles fotovoltaicos con lo cual para futuros trabajos se debe de estudiar una función con todos los costes o ingresos que se puedan generar en la instalación fotovoltaica.

\section{Conclusiones}

En este trabajo se ha comprobado que en instalaciones fotovoltaicas aisladas de bombeo directo es preferible la colocación de varias bombas en paralelo para aprovechar mejor los momentos de menor radiación solar aumentando el volumen impulsado anualmente hasta un $27 \%$. Según los incrementos del volumen bombeado el número óptimo de bombas funcionando en paralelo sería de 5. Así mismo, el aumento del volumen bombeado, para el óptimo de 5 bombas, permite en este caso de estudio que el factor de potencia disminuya en un 50,7\%, además de disminuir el coste entre la hipótesis con 1 bomba y la hipótesis de 5 bombas de un 13.3\%.

\section{Agradecimientos}

Este estudio ha sido parcialmente financiado por el proyecto ADAPTAMED (RTI2018-101483-B-I00), financiado por el Ministerio de Ciencia e Innovación de España y con fondos de la UE FEDER

\section{Referencias}

1. Mérida García, A.; González Perea, R.; Camacho Poyato, E.; Montesinos Barrios, P.; Rodríguez Díaz, J.A. Comprehensive sizing methodology of smart photovoltaic irrigation systems, Agric. Water Manag. 229 (2020) 105888. https://doi.org/10.1016/j.agwat.2019.105888.

2. Hamidat, A.; Benyoucef, B.; Hartani, T. Small-scale irrigation with photovoltaic water pumping system in Sahara regions, Renew. Energy. 28 (2003) 1081-1096. https://doi.org/10.1016/S0960-1481(02)00058-7.

3. López-Luque, R.; x Reca, J. R. Martínez, Optimal design of a standalone direct pumping photovoltaic system for deficit irrigation of olive orchards, Appl. Energy. 149 (2015) 13-23. https://doi.org/10.1016/j.apenergy.2015.03.107.

4. Markvart, T.; Castaner, L. Practical Handbook of Photovoltaics: Fundamentals and Applications, Elsevier Science \& Technology, Kidlington, 2003. 